\title{
The Equilibrium States of the Spin-Boson Model
}

\author{
M. Fannes ${ }^{\star}$, B. Nachtergaele ${ }^{\star \star}$ and A. Verbeure \\ Instituut voor Theoretische Fysica, Universiteit Leuven, B-3030 Leuven, Belgium
}

\begin{abstract}
The temperature states of the spin-boson model consisting of a two-level atom in a Bose field are studied. It is proved that for all temperatures there exists a unique solution, hence there is no spontaneous reflection symmetry breaking.
\end{abstract}

\section{Introduction}

Spin-boson models are very popular in solid state physics, quantum chemistry as well as in quantum tunneling. A fairly good introduction to the physics can be found in [1].

Here we are particularly interested in the following model:

$$
H=\int d k \varepsilon(k) a_{k}^{+} a_{k}+\sigma_{3} \int d k \lambda(k)\left(a_{k}^{+}+a_{k}\right)+\mu \sigma_{1}
$$

describing a two-level atom in a boson field. On the basis of physical arguments one assumes that the following conditions are satisfied:

$$
\int d k \lambda(k)^{2}<\infty, \quad \int d k \frac{\lambda(k)^{2}}{\varepsilon(k)}<\infty ; \quad \varepsilon(k) \simeq|k| .
$$

In this work we study the temperature states of this model in a rigorous way. The ground state problem will be kept for a future occasion. The main aspect we search for is whether or not there is spontaneous symmetry breaking of the reflection symmetry: $\sigma_{3} \rightarrow-\sigma_{3}, a_{k} \rightarrow-a_{k}$. Usual techniques for proving the absence of symmetry breaking are not applicable because the group is finite.

As far as we know there exist only a few rigorous results for this model. In [2] one discusses some results on the spectrum of the proposed Hamiltonian, in [3] a thorough analysis is made of a finite mode approximation of the Hamiltonian. In particular the Hartree-Fock solutions are found to show breaking of the symmetry under the condition $\mu<2 \int\left(\lambda(k)^{2} / \varepsilon(k)\right) d k$. In [4] the ground state of the model is analyzed. By functional integration techniques it is shown that no

$\star \quad$ Bevoegdverklaard Navorser N.F.W.O. Belgium

$\star \star$ Onderzoeker I.I.K.W. Belgium 
spontaneous symmetry breaking appears if $\lambda / \varepsilon$ is square integrable (in fact in $k=0$ ). Otherwise if the coupling $\lambda$ is large enough there is symmetry breaking.

Our contribution consists in the computation of all temperature states, and hence in actually solving the problem for $T>0$. The method we use consists in considering the term $\mu \sigma_{1}$ as a perturbation. This point of view was already presented in [2] and [3]. Then we work towards a rigorous formulation of the KMS-equation (equilibrium conditions) for the so-called unperturbed model, and we are able to solve it completely. Finding the solution relies on a detailed study of the representations of the spin-Boson algebra. Then the perturbation theory is applied at the level of the cyclic vector of the unperturbed system.

From the Hartree-Fock computations and the spectral perturbation theory it could be guessed that symmetry breaking occurs for a coupling which is strong enough. Surprisingly enough it turns out that there is never spontaneous breaking of the symmetry, irrespective of the strength of the coupling constant. In particular we prove that for all positive temperatures there is a unique equilibrium state. We work out the model in one dimension. Our analysis is not complete for higher dimensions. There might be a macroscopic occupation of the ground state $(k=0)$ for the Boson field, destroying the unicity of the solution in two and more dimensions. However we are not interested in the symmetry breaking due to Bose condensation.

\section{The Model}

We start with the CCR-algebra $\Delta\left(\mathscr{H}_{0}\right)$ built on a test function space $\mathscr{H}_{0}$ which is a dense subspace of $L^{2}(\mathbb{R})$. A reasonable choice is $\mathscr{H}_{0}=C_{0}(\mathbb{R})$, the continuous complex functions of compact support on $\mathbb{R}$ vanishing on a neighborhood of zero. As usual we consider the CCR- $C^{*}$-algebra $\Delta\left(\mathscr{H}_{0}\right)$ generated by the Weyl operators

$$
W(f)=\exp i\left(a(f)+a^{+}(f)\right) ; \quad f \in \mathscr{H}_{0},
$$

where $a^{+}(f)$ and $a(f)$ are the usual Fock creation and annihilation operators, which satisfy the product rule

$$
W(f) W(g)=W(f+g) \exp -i \operatorname{Im}(f, g) .
$$

The algebra of observables of the system is then the $C^{*}$-algebra

$$
\mathscr{B}=\Delta\left(\mathscr{H}_{0}\right) \otimes M_{2},
$$

the unique tensor product of $\Delta\left(\mathscr{H}_{0}\right)$ and the $2 \times 2$ complex matrices.

A general element of $\mathscr{B}$ is of the form

$$
X=\left(\begin{array}{ll}
X_{11} & X_{12} \\
X_{21} & X_{22}
\end{array}\right) ; \quad X_{i j} \in \Delta\left(\mathscr{H}_{0}\right)
$$

and a state $\omega$ of $\mathscr{B}$ can be described by a set $\left\{\omega_{i j} \mid i, j=1,2\right\}$ of linear functionals of $\Delta\left(H_{0}\right)$. A useful notation is the following:

$$
\omega=\left(\begin{array}{ll}
\omega_{11} & \omega_{21} \\
\omega_{12} & \omega_{22}
\end{array}\right)
$$


then

$$
\omega: X \rightarrow \omega(X)=\sum_{i, j=1}^{2} \omega_{i j}\left(X_{i j}\right)
$$

By normalization and positivity $\omega$ is a state of $\mathscr{B}$ if and only if the functionals $\omega_{i j}$ satisfy

$$
\begin{gathered}
\omega_{11}(1)+\omega_{22}(1)=1, \omega_{i i}\left(x^{*} x\right) \geqq 0 ; \quad i=1,2 \\
\left|\omega_{12}\left(x^{*} y\right)\right|^{2} \leqq \omega_{11}\left(x^{*} x\right) \omega_{22}\left(y^{*} y\right), \\
\overline{\omega_{12}(x)}=\omega_{21}\left(x^{*}\right)
\end{gathered}
$$

for all $x, y \in \Delta\left(\mathscr{H}_{0}\right)$. For the purpose of the study of the model under consideration we will restrict our attention to the class of states that satisfy the following conditions:

(a) Regularity of the states, i.e. for all $f, g \in \mathscr{H}_{0}$ the map

$$
z \in \mathbb{R} \rightarrow \omega_{i j}(W(f+z g))
$$

is analytic. This condition implies the existence of correlation functions

$$
\omega_{i j}\left(a^{\#}\left(f_{1}\right) \cdots a^{\#}\left(f_{n}\right)\right), \quad f_{i} \in \mathscr{H}_{0}
$$

where $a^{\#}$ stands for $a$ or $a^{+}$.

(b) Continuity of the correlation functions: define the Hilbert space $\mathscr{H}$ as the closure of $\mathscr{H}_{0}$ for the scalar product

$$
(f, g)_{\sim}=\int\left(1+\frac{1}{|k|}\right) \bar{f}(k) g(k) d k .
$$

Then we suppose that the states $\omega_{i i}$ satisfy

$$
\left|\omega_{i i}\left(a^{+}\left(f_{1}\right) \cdots a^{+}\left(f_{n}\right) a\left(g_{1}\right) \cdots a\left(g_{m}\right)\right)\right|^{2} \leqq C^{n+m} n ! m ! \prod_{j=1}^{n}\left\|f_{j}\right\|_{\sim}^{2} \prod_{j=1}^{m}\left\|g_{j}\right\|_{\sim}^{2}
$$

for $f_{j}, g_{j} \in \mathscr{H}_{0}$.

For any state $\omega$ of $\mathscr{B}$ we consider its GNS-triplet $\left(\mathscr{H}_{\omega}, \pi_{\omega}, \Omega_{\omega}\right)$. For notational convenience we identify the algebra and the representation $\left(x=\pi_{\omega}(x)\right.$ for $\left.x \in \mathscr{B}\right)$ if this is clear from the context, and we denote by $\mathscr{B}^{\prime \prime}$ the von Neumann algebra generated by $\mathscr{B}$. Because of the continuity conditions (a) and (b), $\mathscr{B}^{\prime \prime}$ contains the strong limits of $W(f) \otimes 1, f \in \mathscr{H}_{0}$, which again are Weyl operators in that they satisfy the relation (2). This is the meaning of the operators $W(f) \otimes A$ with $f \in \mathscr{H}, A \in M_{2}$. The same reasoning holds true for creation and annihilation operators

$$
a^{\#}(f) \otimes A
$$

with $f \in \mathscr{H}$, as operators on $\mathscr{H}_{\omega}$ affiliated to $\mathscr{B}^{\prime \prime}$. First we describe the evolution $\alpha_{t}^{0}$ on the von Neumann algebra $\mathscr{B}^{\prime \prime}$. Denote by $\sigma_{i}, i=1,2,3$, the Pauli-matrices 
and define for $\varepsilon(k)=|k|$ the maps:

$$
\begin{aligned}
\alpha_{t}^{0}\left(\sigma_{1}\right)= & \frac{1}{2}\left\{W\left(\frac{2 i \lambda}{\varepsilon}\left(e^{i t \varepsilon}-1\right)\right)\left(\sigma_{1}-i \sigma_{2}\right)\right. \\
& \left.+W\left(\frac{2 i \lambda}{\varepsilon}\left(1-e^{i t \varepsilon}\right)\right)\left(\sigma_{1}+i \sigma_{2}\right)\right\}, \\
\alpha_{t}^{0}\left(\sigma_{2}\right)= & \frac{1}{2}\left\{W\left(\frac{2 i \lambda}{\varepsilon}\left(e^{i t \varepsilon}-1\right)\right)\left(\sigma_{2}+i \sigma_{1}\right)\right. \\
& \left.+W\left(\frac{2 i \lambda}{\varepsilon}\left(1-e^{i t \varepsilon}\right)\right)\left(\sigma_{2}-i \sigma_{1}\right)\right\}, \\
\alpha_{t}^{0}\left(\sigma_{3}\right)= & \sigma_{3}, \\
\alpha_{t}^{0}(W(f))= & W\left(e^{i \varepsilon t} f\right) \exp \left\{2 i \sigma_{3} \operatorname{Re}\left(\frac{\lambda}{\varepsilon},\left(e^{i t \varepsilon}-1\right) f\right)\right\}, \quad f \in \mathscr{H} .
\end{aligned}
$$

(c) Existence of the dynamics: we assume that the dynamics $\left\{\alpha_{t}^{0} \mid t \in \mathbb{R}\right\}$ extends to a weakly continuous one-parameter group of *automorphisms of $\mathscr{B}^{\prime \prime}$.

Hence the one-parameter group $\left\{\alpha_{t}^{0} \mid t \in \mathbb{R}\right\}$ defines an infinitesimal generator $\delta_{0}: \alpha_{t}^{0}=\exp i t \delta_{0}$ such that $\delta_{0}$ is formally given by:

$$
\delta_{0}=\left[H_{0}, \cdot\right],
$$

where $H_{0}$ is given by

$$
H_{0}=\int d k \varepsilon(k) a_{k}^{+} a_{k}+\sigma_{3}\left(a(\lambda)+a^{+}(\lambda)\right) .
$$

We now define the full model using the Dyson expansion: for all $x \in \mathscr{B}^{\prime \prime}$ :

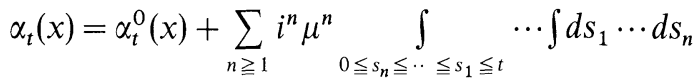

$$
\begin{aligned}
& \cdot\left[\alpha_{s_{n}}^{0}\left(\sigma_{1}\right),\left[\alpha_{s_{n-1}}^{0}\left(\sigma_{1}\right), \cdots\left[\alpha_{s_{1}}^{0}\left(\sigma_{1}\right), \alpha_{t}^{0}(x)\right] \cdots\right]\right.
\end{aligned}
$$

for $t \geqq 0$, and a similar expansion for $t<0$. As the perturbation $\left[\mu \sigma_{1}, \cdot\right]$ is a bounded derivation of $\mathscr{B}^{\prime \prime}$ the series is uniformly convergent and defines a weak *-continuous group of *-automorphisms of $\mathscr{B}^{\prime \prime}$. The infinitesimal generator $\delta$ of the group is formally given by

$$
\delta=[H, \cdot]
$$

where

$$
H=H_{0}+\mu \sigma_{1} .
$$

Remark that by formulae (4) and (5) we arrived at a rigorous definition of the dynamics of the model on the appropriate $C^{*}$-algebra of observables taking into account the conditions

$$
\int \lambda(k)^{2} d k<\infty, \quad \int \frac{\lambda(k)^{2}}{\varepsilon(k)} d k<\infty
$$




\section{Equilibrium States}

We are interested in the equilibrium states at fixed inverse temperature $\beta \geqq 0$ for the full dynamics $\left\{\alpha_{t} \mid t \in \mathbb{R}\right\}$, defined in (5). The strategy consists in constructing the equilibrium states for the unperturbed dynamics $\alpha_{t}^{0}$, then we use the known stability properties of KMS-states for bounded perturbations to obtain the equilibrium states of the full dynamics.

For any state $\omega$ satisfying (a)-(c), we define $\omega$ to be a $\left(\alpha^{0}, \beta\right)$-KMS-state if for all $x, y \in \mathscr{B}_{\alpha^{0}}^{\prime \prime}$, a weakly dense $\alpha^{0}$-invariant subalgebra of $\mathscr{B}^{\prime \prime}$, holds [6]:

$$
\omega\left(x \alpha_{i \beta}^{0}(y)\right)=\omega(y x) .
$$

We prove first that this equilibrium condition has a unique solution for the unperturbed evolution $\alpha^{0}$.

Theorem 3.1. There exists a unique $\left(\alpha^{0}, \beta\right)$-KMS-state $\omega_{\beta}^{0}$ of $\mathscr{B}$ satisfying conditions (a)-(c). Using the notation (3), it is given by

$$
\omega_{\beta}^{0}=\left(\begin{array}{cc}
\frac{1}{2} \omega_{+} & 0 \\
0 & \frac{1}{2} \omega_{-}
\end{array}\right),
$$

where $\omega_{ \pm}$are the states of the CCR-algebra $\Delta\left(\mathscr{H}_{0}\right)$ given by:

$$
\omega_{ \pm}(W(f))=\exp \left\{ \pm 2 i \operatorname{Im}\left(\frac{i \lambda}{\varepsilon}, f\right)-\frac{1}{2}\left(f, \operatorname{coth} \frac{\beta \varepsilon}{2} f\right)\right\} .
$$

In order to prove this theorem we proceed in a number of steps:

Lemma 3.2. If $\omega_{\beta}^{0}$ is a $\left(\alpha^{0}, \beta\right)$-KMS-state, then it is of the form:

$$
\omega_{\eta}=\left(\begin{array}{cc}
\eta \omega_{+} & 0 \\
0 & (1-\eta) \omega_{-}
\end{array}\right)
$$

where $\omega_{ \pm}$are given by $(8), \eta \in[0,1]$.

Proof. First we prove that the off-diagonal components of $\omega_{\beta}^{0}$ vanish. Therefore apply (6) with $x=\sigma_{1} W(f)$ and $y=\sigma_{3}$. Using (4.c) one gets:

$$
\omega_{\beta}^{0}\left(\sigma_{1} W(f) \alpha_{i \beta}^{0}\left(\sigma_{3}\right)\right)=\omega_{\beta}^{0}\left(\sigma_{3} \sigma_{1} W(f)\right)
$$

and

$$
\omega_{\beta}^{0}\left(\sigma_{1} \sigma_{3} W(f)\right)=\omega_{\beta}^{0}\left(\sigma_{3} \sigma_{1} W(f)\right)
$$

or

$$
\omega_{\beta}^{0}\left(\sigma_{2} W(f)\right)=0
$$

Analogously:

$$
\omega_{\beta}^{0}\left(\sigma_{1} W(f)\right)=0 .
$$

Therefore $\omega_{\beta}^{0}$ is of the form

$$
\omega_{\beta}^{0}=\left(\begin{array}{cc}
\omega_{1} & 0 \\
0 & \omega_{2}
\end{array}\right)
$$


where

$$
\begin{array}{ll}
\omega_{1}(W(f))=\omega_{\beta}^{0}\left(\frac{1}{2}\left(1+\sigma_{3}\right) W(f)\right) ; & f \in \mathscr{H}_{0}, \\
\omega_{2}(W(f))=\omega_{\beta}^{0}\left(\frac{1}{2}\left(1-\sigma_{3}\right) W(f)\right) ; & f \in \mathscr{H}_{0} .
\end{array}
$$

Define the automorphism groups $\left\{\alpha_{t}^{ \pm} \mid t \in \mathbb{R}\right\}$ of $\Delta\left(\mathscr{H}_{0}\right)$ by

$$
\alpha_{t}^{ \pm}(W(f))=W\left(e^{i \varepsilon t} f\right) \exp \left\{ \pm 2 i \operatorname{Re}\left(\frac{\lambda}{\varepsilon},\left(e^{i \varepsilon t}-1\right) f\right)\right\}
$$

From (4.c) one has

$$
\frac{1}{2}\left(1 \pm \sigma_{3}\right) \alpha_{t}^{ \pm}(W(f))=\frac{1}{2}\left(1 \pm \sigma_{3}\right) \alpha_{t}^{0}(W(f)) .
$$

Remark that for all $f, g \in \mathscr{H}_{0}$,

$$
\begin{aligned}
\omega_{1}\left(W(f) \alpha_{i \beta}^{+} W(g)\right) & =\omega_{\beta}^{0}\left(W(f) \frac{1}{2}\left(1+\sigma_{3}\right) \alpha_{i \beta}^{+}(W(g))\right) \\
& =\omega_{\beta}^{0}\left(W(f) \frac{1}{2}\left(1+\sigma_{3}\right) \alpha_{i \beta}^{0}(W(g))\right) \\
& =\omega_{\beta}^{0}\left(W(g) W(f) \frac{1}{2}\left(1+\sigma_{3}\right)\right) \\
& =\omega_{1}(W(g) W(f)),
\end{aligned}
$$

and similarly $\omega_{2}$ is a $\left(\alpha^{-}, \beta\right)$-KMS-state up to normalization.

The automorphisms $\alpha^{ \pm}$are up to a displacement the free Bose-gas automorphisms. Under our general conditions $\lambda^{2} / \varepsilon$ and $\lambda^{2} \in L^{1}(\mathbb{R}),(\mathrm{a})-(\mathrm{c})$, they yield the unique KMS-states $\omega^{ \pm}$given by (8) [5]. Hence there exists a $\eta \in[0,1]$ such that

$$
\omega_{1}=\eta \omega_{+}, \quad \omega_{2}=(1-\eta) \omega_{-},
$$

where $\eta=\omega_{1}(\mathbb{1})$.

The lemma shows that all solutions of (6) are of the type given by (9) with $\eta \in[0,1]$. We have to prove that there exists only one solution, namely corresponding to the value $\eta=\frac{1}{2}$. Therefore define the reflection symmetry automorphism $\tau$ on $\mathscr{B}$ by the following relations:

$$
\begin{aligned}
\tau\left(\sigma_{1}\right) & =\sigma_{1}, \quad \tau\left(\sigma_{2}\right)=-\sigma_{2}, \quad \tau\left(\sigma_{3}\right)=-\sigma_{3}, \\
\tau(W(f)) & =W(-f), \quad f \in \mathscr{H},
\end{aligned}
$$

and remark that $\omega_{\eta}^{\circ} \tau=\omega_{1-\eta}$.

The state $\omega_{\eta}$ with $\eta=\frac{1}{2}$ is then precisely the unique $\tau$-invariant state in (9).

Now the strategy to finish the proof of Theorem 3.1 consists in proving that any $\left(\alpha^{0}, \beta\right)$-KMS-state is $\tau$-invariant. In order to obtain this statement we prove that under the general conditions on the model any state of the class (9) is a factor state. This will be a consequence of a more general study of the representations induced by states of that type, which we present in the appendix (Sect. 4). Then we remark that $\tau$ is an implementable automorphism, commuting with the time evolution, implying that any solution of (6) is $\tau$-invariant which by the argument of above implies $\eta=\frac{1}{2}$. 
Lemma 3.3. Under the conditions

$$
\int d k \frac{\lambda(k)^{2}}{\varepsilon(k)}<\infty, \quad \int d k \lambda(k)^{2}<\infty,
$$

the states $\omega_{\eta}$ of $\mathscr{B}=\Delta\left(\mathscr{H}_{0}\right) \otimes M_{2}$

$$
\left(\begin{array}{cc}
\eta \omega_{+} & 0 \\
0 & (1-\eta) \omega_{-}
\end{array}\right)
$$

with $\eta \in[0,1]$ given in (9) are factor states.

Proof. If $\eta=0$ or 1 one gets the states $\omega_{+}$and $\omega_{-}$which are well known to be factor states [7].

From Proposition $4.3, \omega_{+}$and $\omega_{-}$are quasi-equivalent states of $\Delta\left(\mathscr{H}_{0}\right)$ because

$$
\begin{aligned}
\left|\left(\frac{\lambda}{\varepsilon}, f\right)\right|^{2} & =\left.\left(\frac{\lambda}{\varepsilon} \tanh ^{1 / 2}\left(\frac{\beta \varepsilon}{2}\right), \operatorname{coth}^{1 / 2}\left(\frac{\beta \varepsilon}{2}\right) f\right)\right|^{2} \\
& \leqq\left(f, \operatorname{coth}\left(\frac{\beta \varepsilon}{2}\right) f\right)\left(\frac{\lambda}{\varepsilon}, \tanh \left(\frac{\beta \varepsilon}{2}\right) \frac{\lambda}{\varepsilon}\right)
\end{aligned}
$$

and $\lambda^{2} \varepsilon^{-2} \tanh \left(\frac{1}{2} \beta \varepsilon\right) \in L^{1}(\mathbb{R})$ because $\int d k \lambda(k)^{2} \varepsilon(k)^{-1}<\infty$, fulfilling the conditions of Proposition 4.3 with $A=\operatorname{coth}\left(\frac{1}{2} \beta \varepsilon\right)$. Hence there exists a non-zero intertwining unitary operator between $\omega_{+}$and $\omega_{-}$.

As $\omega_{+}$and $\omega_{-}$are factor states it follows now from Proposition 4.2 that also for $\eta \in(0,1)$ the states $(11)$ are factor states.

Lemma 3.4. Denote again by $\mathscr{B}^{\prime \prime}$ the von Neumann algebra of the state $\omega_{\eta}$ of $\mathscr{B}$, $\eta \in[0,1]$. Under the conditions $\int d k \lambda(k)^{2} \varepsilon(k)^{-1}<\infty$ and $\int d k \lambda(k)^{2}<\infty$ the automorphism $\tau$ of $\mathscr{B}$ defined in (10), extends to a unitary implementable automorphism of $\mathscr{B}^{\prime \prime}$.

Proof. Let $\left(\mathscr{H}_{+}, \pi_{+}, \Omega_{+}\right)$denote the GNS-representation of $\omega_{+}$. By the conditions on $\lambda$ we can extract from Proposition 4.3 and the proof of Lemma 3.3 the existence of a unitary operator $U \in \mathscr{B}\left(\mathscr{H}_{+}\right)$, which satisfies

$$
\omega_{-}(x)=\left\langle U \Omega_{+} \mid \pi_{+}(x) U \Omega_{+}\right\rangle, \quad x \in \Delta\left(\mathscr{H}_{0}\right) .
$$

Consider now the mapping

$$
V: \pi(W(f)) \Omega_{+} \rightarrow \pi_{+}(W(-f)) U \Omega_{+}, \quad f \in \mathscr{H}_{0},
$$

then

$$
\begin{aligned}
\left\langle\pi_{+}\right. & \left(W\left(-f_{1}\right)\right) U \Omega_{+}\left|\pi_{+}\left(W\left(-f_{2}\right)\right) U \Omega_{+}\right\rangle \\
& =\left\langle U \Omega_{+} \mid \pi_{+}\left(W\left(f_{1}\right) W\left(-f_{2}\right)\right) U \Omega_{+}\right\rangle \\
& =\omega_{-}\left(W\left(f_{1}\right) W\left(-f_{2}\right)\right) \\
& =\omega_{+}\left(W\left(-f_{1}\right) W\left(f_{2}\right)\right) \\
& =\left\langle\pi_{+}\left(W\left(f_{1}\right)\right) \Omega_{+} \mid \pi_{+}\left(W\left(f_{2}\right)\right) \Omega_{+}\right\rangle .
\end{aligned}
$$

Therefore $V$ extends to an isometry of $\mathscr{H}_{+}$and as $W(f) \rightarrow W(-f)$ defines an 
automorphism of $\Delta\left(\mathscr{H}_{0}\right)$ it is easy to check that $V$ is unitary. Furthermore

$$
\pi_{+}(W(-f))=V \pi_{+}(W(f)) V^{*}, \quad f \in \mathscr{H}_{0} .
$$

Therefore by Proposition 4.2 and using $\pi_{+}=\pi_{-}$and $\mathscr{H}_{+}=\mathscr{H}_{-}$, one concludes that

$$
\mathscr{B}^{\prime \prime}=\left\{\left(\begin{array}{cccc}
a_{1} & 0 & a_{2} & 0 \\
0 & a_{4} & 0 & a_{3} \\
a_{3} & 0 & a_{4} & 0 \\
0 & a_{2} & 0 & a_{1}
\end{array}\right) \mid a_{i} \in \pi_{+}\left(\Delta\left(\mathscr{H}_{0}\right)\right)^{\prime \prime}\right\} .
$$

Now it is easily checked that the automorphism $\tau$ is implemented by the unitary

$$
Q=\left(\begin{array}{cccc}
V & 0 & 0 & 0 \\
0 & V & 0 & 0 \\
0 & 0 & V & 0 \\
0 & 0 & 0 & V
\end{array}\right) \pi_{\eta}\left(\sigma_{1}\right)
$$

where $\pi_{\eta}$ is the representation induced by $\omega_{\eta}$.

Combining the arguments of above we now obtain:

Proof of Theorem 3.1. By Lemma 3.4 the automorphism $\tau$ of (10) extends to an implementable automorphism of the von Neumann algebra $\mathscr{B}^{\prime \prime}$ of $\omega_{\eta}$ and we again denote this automorphism by $\tau$. Clearly $\omega_{\eta}{ }^{\circ} \tau=\omega_{1-\eta}$.

From the formulas (4.a)-(4.d) it follows furthermore that $\alpha_{t}^{0} \circ \tau=\tau \circ \alpha_{t}^{0}, t \in \mathbb{R}$. Let now $\omega_{\beta}^{0}=\omega_{\eta}$ be an $\left(\alpha_{t}^{0}, \beta\right)$-KMS-state (Lemma 3.2), then also $\omega_{1-\eta}$ has to be $\left(\alpha_{t}^{0}, \beta\right)$-KMS and $\omega_{1-\eta}$ is a normal state of $\mathscr{B}^{\prime \prime}$ by the arguments of above. As by Lemma $3.3 \mathscr{B}^{\prime \prime}$ is a factor we conclude by [6, Prop. 5.3.29] that $\omega_{\eta}=\omega_{1-\eta}$ or equivalently that $\eta=\frac{1}{2}$. This proves that there exists a unique $\left(\alpha_{t}^{0}, \beta\right)$-KMS-state of $\mathscr{B}$ explicitly given by formula (7).

The equilibrium states of the full model can now be computed by a perturbation of the equlibrium states of the solvable model $\left\{\alpha_{t}^{0} \mid t \in \mathbb{R}\right\}$. In order to do this we need the perturbation technique on von Neumann algebras developed in the context of stability theory for KMS-states.

Theorem 3.5. Under the conditions $\int d k \lambda^{2} \varepsilon^{-1}<\infty$ and $\int d k \lambda^{2}<\infty$ the full model defined in (5) admits for every positive $\beta$ a unique $(\alpha, \beta)$-KMS-state $\omega_{\beta}$ which satisfies (a)-(c). Furthermore $\omega_{\beta}$ is normal with respect to the unique $\left(\alpha^{0}, \beta\right)$-KMS-state $\omega_{\beta}^{0}$ and is given by the following strongly convergent perturbation expansion:

Let $\left(\mathscr{H}_{0}, \pi_{0}, \Omega_{0}\right)$ be the GNS triplet of $\omega_{\beta}^{0}$, then

$$
\omega_{\beta}(x)=\frac{\left\langle\Omega \mid \pi_{0}(x) \Omega\right\rangle}{\|\Omega\|^{2}}, \quad x \in \mathscr{B},
$$

where

$$
\Omega=\Omega_{0}+\sum_{n \geqq 1}(-\beta \mu)^{n} \int_{0 \leqq s_{n} \leqq . . \leqq s_{1} \leqq 1 / 2} d s_{1} \cdots d s_{n} \alpha_{i \beta s_{n}}^{0}\left(\sigma_{1}\right) \cdots \alpha_{i \beta s_{1}}^{0}\left(\sigma_{1}\right) \Omega_{0} .
$$

It follows that $\omega_{\beta}$ is $\tau$-invariant where $\tau$ is defined in (10), in particular $\omega_{\beta}\left(\sigma_{3}\right)=0$. 
Proof. Using [6, Theorem 5.4.4] one constructs a KMS-state $\omega_{\beta}$ for a perturbed dynamics $\alpha$ (5) from the unperturbed one $\alpha^{0}$ (4). The full dynamics $\alpha$ is obtained by adding a bounded operator $\mu \sigma_{1}$ to the Hamiltonian.

The state $\omega_{\beta}$ is given by its cyclic vector which is constructed in terms of a series expansion (11). As the unperturbed state $\omega_{\beta}^{0}$ is a factor state, $\omega_{\beta}$ is the unique $(\alpha, \beta)$-KMS-state which is normal with respect to $\omega_{\beta}^{0}$. As $\omega_{\beta}^{0}$ is unique (Theorem 3.1) also $\omega_{\beta}$ is unique. In particular $\omega_{\beta}$ is $\tau$-invariant.

\section{Appendix}

A. Consider the $C^{*}$-algebra $\mathscr{B}=\mathscr{A} \otimes M_{2}$, where $\mathscr{A}$ is a $C^{*}$-algebra. Hence a general element of $\mathscr{B}$ can be written in the matrix form

$$
x \in \mathscr{B}: x=\left(\begin{array}{ll}
x_{11} & x_{12} \\
x_{21} & x_{22}
\end{array}\right), \quad x_{i j} \in \mathscr{A} .
$$

Suppose that $\omega_{1}$ and $\omega_{2}$ are states of $\mathscr{A}$, then

$$
\omega_{\eta}: x \in \mathscr{B} \rightarrow \omega_{\eta}(x)=\eta \omega_{1}\left(x_{11}\right)+(1-\eta) \omega_{2}\left(x_{22}\right)
$$

is a state of $\mathscr{B}$ for all $\eta \in[0,1]$. Let $\left(\mathscr{H}_{i}, \pi_{i}, \Omega_{i}\right), i=1,2$ be the GNS-representations of $\mathscr{A}$ defined by the states $\omega_{i}, i=1,2$, then we have:

Proposition 4.1. For all $\eta \in(0,1), \omega_{\eta}$ induces the GNS-representation $\left(\mathscr{H}_{\eta}, \pi_{\eta}, \Omega_{\eta}\right)$, where

$$
\begin{aligned}
\mathscr{H}_{\eta} & =\mathscr{H}_{1} \oplus \mathscr{H}_{2} \oplus \mathscr{H}_{1} \oplus \mathscr{H}_{2}, \\
\Omega_{\eta} & =\left(\begin{array}{c}
\eta^{1 / 2} \Omega_{1} \\
(1-\eta)^{1 / 2} \Omega_{2} \\
0 \\
0
\end{array}\right) \in \mathscr{H}_{\eta}, \\
\pi_{\eta}(x) & =\left(\begin{array}{cccc}
\pi_{1}\left(x_{11}\right) & 0 & \pi_{1}\left(x_{12}\right) & 0 \\
0 & \pi_{2}\left(x_{22}\right) & 0 & \pi_{2}\left(x_{21}\right) \\
\pi_{1}\left(x_{21}\right) & 0 & \pi_{1}\left(x_{22}\right) & 0 \\
0 & \pi_{2}\left(x_{12}\right) & 0 & \pi_{2}\left(x_{11}\right)
\end{array}\right) .
\end{aligned}
$$

Proof. A straightforward computation yields

$$
\begin{aligned}
\left\langle\Omega_{\eta} \mid \pi_{\eta}(x) \Omega_{\eta}\right\rangle & =\omega(x), \quad x \in \mathscr{B}, \\
\pi_{\eta}(x) \pi_{\eta}(y) & =\pi_{\eta}(x y), \quad x, y \in \mathscr{B},
\end{aligned}
$$

and the cyclicity of $\Omega_{\eta}$ for $\pi_{\eta}$ follows from $0<\eta<1$.

Denote by $\mathscr{M}_{i}=\pi_{i}(\mathscr{A})^{\prime \prime}$ the von Neumann algebras defined by the states $\omega_{i}$ of $\mathscr{A}$ for $i=1,2$, by $\mathscr{M}_{i}^{\prime}$ the commutants of the $\mathscr{M}_{i}$ and by $\mathscr{T}$ the set of operators from $\mathscr{H}_{1}$ to $\mathscr{H}_{2}$, intertwining the representations $\pi_{1}$ and $\pi_{2}$, i.e. if $t \in \mathscr{T}$ then $t \in \mathscr{B}\left(\mathscr{H}_{1}, \mathscr{H}_{2}\right)$ such that for all $y \in \mathscr{A}$ holds $t \pi_{1}(y)=\pi_{2}(y) t$. In the next proposition we characterize the von Neumann algebra $\mathscr{M}_{\eta}$ of the state $\omega_{\eta}$. 
Proposition 4.2. With the notations of above we have that $\mathscr{M}_{\eta}^{\prime}$ is the set of operators in $B\left(\mathscr{H}_{\eta}\right)$ :

$$
\left(\begin{array}{cccc}
y_{1}^{\prime} & 0 & 0 & t_{1}^{*} \\
0 & y_{2}^{\prime} & t_{2} & 0 \\
0 & t_{1}^{*} & y_{1}^{\prime} & 0 \\
t_{2} & 0 & 0 & y_{2}^{\prime}
\end{array}\right),
$$

where $y_{i}^{\prime} \in \mathscr{M}_{i}^{\prime}, t_{i} \in \mathscr{T}(i=1,2)$; furthermore, $\mathscr{M}_{\eta}$ is given $b y$

$$
\left(\begin{array}{cccc}
a_{1} & 0 & a_{2} & 0 \\
0 & b_{4} & 0 & b_{3} \\
a_{3} & 0 & a_{4} & 0 \\
0 & b_{2} & 0 & b_{1}
\end{array}\right),
$$

where $a_{i} \in \mathscr{M}_{1}, b_{i} \in \mathscr{M}_{2}, t a_{i}=b_{i} t, a_{i} t^{*}=t^{*} b_{i}$ for $i=1,2,3,4$ and $t \in \mathscr{T}$.

The center $\mathscr{M}_{\eta} \cap \mathscr{M}_{\eta}^{\prime}$ is then

$$
\left(\begin{array}{cccc}
z_{1} & 0 & 0 & 0 \\
0 & z_{2} & 0 & 0 \\
0 & 0 & z_{1} & 0 \\
0 & 0 & 0 & z_{2}
\end{array}\right)
$$

where $z_{i} \in \mathscr{M}_{i} \cap \mathscr{M}_{i}^{\prime},(i=1,2)$ and satisfy $t z_{1}=z_{2} t$ and $z_{1} t^{*}=t^{*} z_{2}$ for all $t \in \mathscr{T}$.

Proof. Using the matrix representation of Proposition 4.1 one computes explicitly the commutant $\mathscr{M}_{\eta}^{\prime}$ by solving for $X$ the matrix equations

$$
\pi_{\eta}(x) X=X \pi_{\eta}(x) \text { for all } x \in \mathscr{B}
$$

with $X \in \mathscr{B}\left(\mathscr{H}_{\eta}\right)$. The computation of the von Neumann algebra $\mathscr{M}_{\eta}$ is obtained in a similar way. The center $\mathscr{M}_{\eta} \cap \mathscr{M}_{\eta}^{\prime}$ is then immediately recognized.

B. Consider $\mathscr{H}$, a complex Hilbert space with scalar product $(\cdot, \cdot)$, and $A$ a self-adjoint operator on $\mathscr{H}$ such that $A \geqq 1$. Let $\mathscr{H}_{0}$ be a dense subspace of $\mathscr{H}$ contained in the domain of $A^{1 / 2}$ and consider the CCR-C*-algebra $\Delta\left(\mathscr{H}_{0}\right)$. It is well known that for each linear functional $\chi$ on $\mathscr{H}_{0}, \omega_{A, \chi}$ defined by

$$
\omega_{A, \chi}(W(f))=\exp \left\{-\frac{1}{2}(f, A f)+i \operatorname{Im} \chi(f)\right\}
$$

extends to a state of $\Delta\left(\mathscr{H}_{0}\right)$. The GNS-representation of $\omega_{A, \chi}$ is given by:

- the representation space is a subspace of $\mathscr{H}_{F} \otimes \mathscr{H}_{F}$, where $\mathscr{H}_{F}$ is the usual Fock space;

- the representation

$$
\pi_{A, \chi}(W(f))=\exp (i \operatorname{Im} \chi(f)) W\left(\left(\frac{A+1}{2}\right)^{1 / 2} f\right) \otimes W \overline{\left(\left(\frac{A-1}{2}\right)^{1 / 2} f\right)},
$$

where ${ }^{\top}$ means complex conjugation;

- the cyclic vector: $\Omega_{A, \chi}=\Omega_{F} \otimes \Omega_{F}, \Omega_{F}$ is the Fock vacuum. 
It is also well known that $\pi_{A, \chi}$ is a factor representation [7].

Here we are interested in the necessary and sufficient condition in order that two states $\omega_{A, \chi_{1}}$ and $\omega_{A, \chi_{2}}$ are quasi-equivalent. The case $A=1$ and $\chi_{1}=0$ is treated in [8]. In order to formulate the condition we introduce the Hilbert space $\mathscr{H}_{A}=\mathscr{D}\left(A^{1 / 2}\right)$ with norm $\|f\|_{A}=\left\|A^{1 / 2} f\right\|$.

Proposition 4.3. The states $\omega_{A, \chi_{1}}$ and $\omega_{A, \chi_{2}}$ of $\Delta\left(\mathscr{H}_{0}\right)$ are quasi-equivalent if and only if $\chi_{1}-\chi_{2}$ extends continuously to $\mathscr{H}_{A}$. Moreover quasi-equivalence implies unitary equivalence.

Proof. Suppose first that $\chi_{1}-\chi_{2}$ extends continuously to $\mathscr{H}_{A}$, then there exists a $h \in \mathscr{H}_{A}$ such that

$$
\left(\chi_{1}-\chi_{2}\right)(f)=(h, f)_{A}, \quad f \in \mathscr{H}_{A}
$$

Take the element

$$
g=-\frac{A^{1 / 2}}{2}\left(\frac{2 A}{A+1}\right)^{1 / 2} h .
$$

As $h \in \mathscr{D}\left(A^{1 / 2}\right)$ and $(A / A+1)^{1 / 2} \leqq 1$, one has $g \in \mathscr{H}$, clearly $W(g) \otimes \mathbb{1}$ is a unitary operator on the representation space and

$$
\begin{aligned}
\left\langle\boldsymbol{\Omega}_{A, \chi_{1}} \mid W(-g) \otimes 1 \pi_{A, \chi_{1}}(W(f)) W(g) \otimes 1 \Omega_{A, \chi_{1}}\right\rangle \\
\quad=\exp \left\{2 i \operatorname{Im}\left(g,\left(\frac{A+1}{2}\right)^{1 / 2} f\right)\right\} \omega_{A, \chi_{1}}(W(f)) \\
\quad=\exp \left\{2 i \operatorname{Im}\left(g,\left(\frac{A+1}{2}\right)^{1 / 2} f\right)+i \operatorname{Im}\left(\chi_{1}-\chi_{2}\right)(f)\right\} \omega_{A, \chi_{2}}(W(f)) \\
=\omega_{A, \chi_{2}}(W(f)),
\end{aligned}
$$

using (12) and (13). Hence $\omega_{A, \chi_{1}}$ and $\omega_{A, \chi_{2}}$ are quasi-equivalent.

Conversely suppose that $\chi_{1}-\chi_{2}$ does not extend continuously to $\mathscr{H}_{A}$, then there exists a sequence $\left(f_{n}\right)_{n}$ of elements $f_{n}$ in $\mathscr{H}_{0}$, such that $\left\|f_{n}\right\| \leqq(1 / n)$ and $\left|\operatorname{Im}\left(\chi_{1}-\chi_{2}\right)\left(f_{n}\right)\right|=\pi$, and for every $g \in \mathscr{H}_{0}$ one has

$$
\begin{aligned}
\left\|\omega_{A, \chi_{1}}(W(-g) \cdot W(g))-\omega_{A, \chi_{2}}\right\| \geqq & \left|\omega_{A, \chi_{1}}\left(W(-g) W\left(f_{n}\right) W(g)\right)-\omega_{A, \chi_{2}}\left(W\left(f_{n}\right)\right)\right| \\
= & \mid \exp i \operatorname{Im}\left(\left(g, f_{n}\right)+\chi_{1}\left(f_{n}\right)\right) \\
& -\exp i \operatorname{Im} \chi_{2}\left(f_{n}\right) \mid \exp -\frac{1}{2}\left(f_{n}, A f_{n}\right) \\
& \rightarrow 2 \text { if } n \rightarrow \infty .
\end{aligned}
$$

Suppose now that there exists a non-zero intertwining operator $T \in \mathscr{B}\left(h_{2}, h_{1}\right)$ for the representations $\pi_{A, \chi_{2}}$ and $\pi_{A, \chi_{1}}$, such that for all $x \in \Delta\left(\mathscr{H}_{0}\right)$

$$
\pi_{A, \chi_{1}}(x) T=T \pi_{A, \chi_{2}}(x) \text {. }
$$

It follows that $0<T^{*} T \in \pi_{A, \chi_{2}}\left(\Delta\left(\mathscr{H}_{0}\right)\right)^{\prime}$ and that a state $\eta$ of $\Delta\left(\mathscr{H}_{0}\right)$ can be defined by:

$$
\eta(x)=\frac{\left\langle\Omega_{A, \chi_{2}} \mid T^{*} T \pi_{A, \chi_{2}}(x) \Omega_{A, \chi_{2}}\right\rangle}{\left\langle\Omega_{A, \chi_{2}} \mid T^{*} T \Omega_{A, \chi_{2}}\right\rangle}=\frac{\left\langle T \Omega_{A, \chi_{2}}, \pi_{A, \chi_{1}}(x) T \Omega_{A, \chi_{2}}\right\rangle}{\left\|T \Omega_{A, \chi_{2}}\right\|^{2}}
$$


such that

$$
\eta(\cdot) \leqq c \omega_{A, \chi_{2}}(\cdot)
$$

for some $c \in \mathbb{R}^{+}$. From (14) it follows that for any $\varepsilon>0$ one can find a selfadjoint $x$, $\|x\| \leqq 1$, such that

\section{Hence}

$$
\omega_{A, \chi_{1}}(W(-g) x W(g))-\omega_{A, \chi_{2}}(x) \geqq 2-\varepsilon .
$$

and with (15)

Now it follows that

$$
\omega_{A, \chi_{1}}(x) \geqq 1-\varepsilon ; \quad \omega_{A, \chi_{2}}(x) \leqq-1+\varepsilon
$$

$$
0 \leqq \eta(1+x) \leqq c \omega_{A, \chi_{2}}(1+x)=c \varepsilon
$$

$$
\left\|\omega_{A, \chi_{1}}(W(-g) \cdot W(g))-\eta(\cdot)\right\|=2 .
$$

For any two normalized vectors $\phi_{1}$ and $\phi_{2}$ in a Hilbertspace one has in general

$$
\left\|\left\langle\phi_{1} \mid \cdot \phi_{1}\right\rangle-\left\langle\phi_{2} \mid \cdot \phi_{2}\right\rangle\right\| \leqq 2\left(1-\left|\left\langle\phi_{1} \mid \phi_{2}\right\rangle\right|^{2}\right)^{1 / 2} \text {. }
$$

Take now $\phi_{1}=\pi_{A, \chi_{1}}(W(g)) \Omega_{A, \chi_{1}}$ and $\phi_{2}=T \Omega_{A, \chi_{2}}$, then from (16)

$$
\left\langle T \Omega_{A, \chi_{2}}, \pi_{A, \chi_{1}}(W(g)) \Omega_{A, \chi_{1}}\right\rangle=0
$$

As $\Omega_{A, \chi_{1}}$ is cyclic for $\pi_{A, \chi_{1}}\left(\Delta\left(\mathscr{H}_{0}\right)\right)$ and $\Omega_{A, \chi_{2}}$ is separating for $\pi_{A, \chi_{2}}\left(\Delta\left(\mathscr{H}_{0}\right)\right)^{\prime}$, we can conclude that $T=0$. Therefore $\omega_{A, \chi_{1}}$ and $\omega_{A, \chi_{2}}$ cannot be quasiequivalent.

Acknowledgement. One of us (A.V.) thanks Herbert Spohn for bringing the model to his attention.

\section{References}

1. Leggett, A. J., Chakravarty, S., Dorsey, A. T., Fisher, M. P. A., Garg, Anupam, Zwerger, W.: Dynamics of the dissipative two-state system. Rev. Mod. Phys. 180

2. Davies, E. B.: Symmetry breaking for molecular open systems. Ann. Inst. H. Poincaré A35, 149 (1981)

3. Pfeifer, P.: Chiral Molecules. Diss. ETH N. 6551, Zürich, 1980

4. Spohn, H., Dümcke, R.: Quantum Tunnfling with dissipation and the Ising model over $\mathbb{R}$. J. Stat. Phys. 41, 389 (1985)

5. Rocca, F., Sirugue, M., Testard, D.: On a class of equilibrium states under the Kubo-MartinSchwinger Condition, II. Bosons. Commun. Math. Phys. 19, 119 (1970)

6. Bratteli, O., Robinson, D. W.: Operator algebras and quantum statistical mechanics, II. Berlin, Heidelberg, New York: Springer 1981

7. Araki, H., Woods, E. J.: Representations of the canonical commutation relations describing a nonrelativistic infinite free Base gas. J. Math. Phys. 4, 637 (1963)

8. Manuceau, J.: Etude de quelques automorphismes de la $C^{*}$-algebra du champ de bosons libres. Ann. Inst. H. Poincaré VIIIA2, 117 (1968)

Communicated by H. Araki

Received May 11, 1987; in revised form August 24, 1987 